\title{
Development of Auto-Stacking Warehouse Truck
}

\author{
Kuo-Hsien Hsia, Ming-Guang Wu, Jun-Nong Lin, Hong-Jie Zhong, and Zh-Yao Zhuang \\ Department of Electrical Engineering, Far-East University, Taiwan \\ Tainan, 74448, Taiwan (R.O.C.) \\ E-mail:khhsia@mail.feu.edu.tw \\ www.feu.edu.tw
}

\begin{abstract}
Warehouse automation is a very important issue for the promotion of traditional industries. For the production of larger and stackable products, it is usually necessary to operate a fork-lifter for the stacking and storage of the products by a skilled person. The general autonomous warehouse-truck does not have the ability of stacking objects. In this paper, we develop a prototype of auto-stacking warehouse-truck that can work without direct operation by a skill person. With command made by an RFID card, the stacker truck can take the packaged product to the warehouse on the prior-planned route and store it in a stacking way in the designated storage area, or deliver the product to the shipping area or into the container from the storage area. It can significantly reduce the manpower requirements of the skilled-person of forklift technician and improve the safety of the warehousing area.
\end{abstract}

Keywords: Warehouse automation, Stacking truck, Autonomous warehouse truck, RFID.

\section{Introduction}

The industrial automation has been developed for more than 60 years. It includes manufacturing automation, warehouse automation, and logistics automation. No matter in which one of these automations, robots always play an extremely important and crucial role in the industrial automation. For the manufacturing industry, object movement is always inevitable in the process of purchasing of raw materials or components, loading of materials, assembling the parts, and packing or shipping the finished products. A conveyor belt is usually used for delivering the components, semi-finished products and finished products in the production line. However, after the product is completed and packaged, it is necessary to use manpower to move the packaged products to the warehouse and stack them to a designated storage location.

Although the automation of manufacturing processes and the automation of manufacturing industries have been able to evolve to unmanned factories, it is usually still necessary to manually drive a forklift truck from factory to warehouse or from warehouse to delivery, to inbound and outbound delivery of the warehouse, etc. to carry out stacking and picking operations. To drive a stacker requires professional training. In Taiwan, a technician license (Technician for fork lift operation) with the Ministry of Labor is required to qualify for driving a stacker. In addition, where people work, they must also cooperate with lighting, air circulation, and various conditions of work safety. Therefore, if it is not necessary to work with manpower, then the cost of lighting and air conditioning can be saved and the space can be used more effectively. Hence many researchers have invested in storage automation research. Many logistics providers also include warehouse automation as one of the most important work items available today.

Giordano et. al considered a matrix-based discrete event control approach for a warehouse [1]. Xie et al use RFID (radio frequency identifier) and wireless sensor network 
in managing a warehouse [2]. In [3], Adinandra et al said that autonomous mobile robots (AMR) have emerged as a means of transportation system in warehouses. Wang et al proposed a positioning system composed of an RFID reader equipped with forklift and terrestrial channels embedded RFID tags to improve warehouse management efficiency [4]. Amazon makes extensive use of robots for product collation and warehouse management and had 45,000 robots across 20 fulfillment centers in the early 2017 [5].

In this paper, we will develop a small warehouse truck with the function of auto-stacking. Here "auto-stacking" means the goods can be stored in stack manner with detection of numbers of stacked goods and it will alarm when the storage area is full.

\section{System Architecture}

In the case of a typical factory, when the goods are manufactured by the production line and packaged, they are usually manually moved to a van and then stacked by a technician of fork-lifter to the warehouse area. When there is a shipping requirement, they will be transported in the same way from the warehouse area to the shipping area for packing or into the container. Different products will be placed in different warehouses or different counter. We assume that on the product or its package, there is an RFID (radio frequency identifier) tag for identification. In addition, our scenario assumes that the warehouse is an unmanned environment and all orders are made in the shipping area or packing area. Because an unmanned storage environment does not need any lighting for vision and air-conditioning, it is energysaving. It also can reduce the possibility of potential danger of injury caused by the devices movement or improper operation since no person is required to work in it. The flow of the goods with RFID tag is shown in Figure 1.

Thus the warehouse truck with the auto-stacking ability should be composed of three parts: the driver part to driving the motors of the truck and the mechanism of the fork-lift device, the control part to command the truck the destination and route, and the sensor part including RFID reader for commanding, sensors for obstacle avoidance and tracking the route, and sensors for the fork of the truck. The system architecture of the auto-stacking warehouse truck is shown in Figure 2.

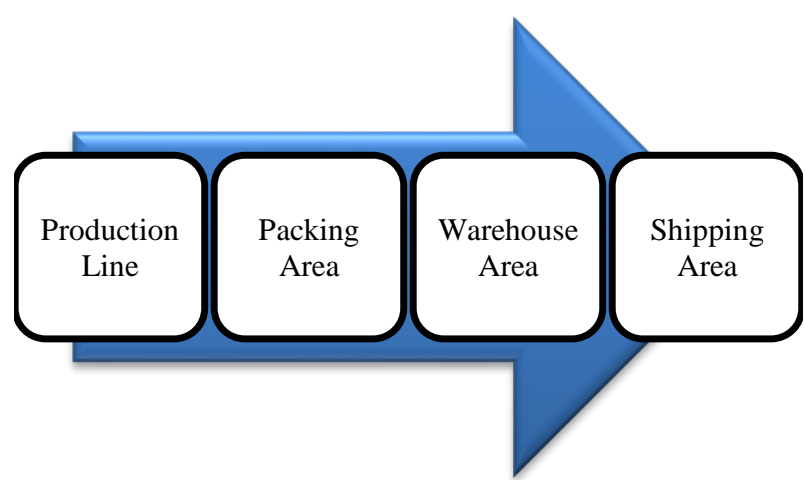

Fig. 1. Flow of goods with RFID tag.

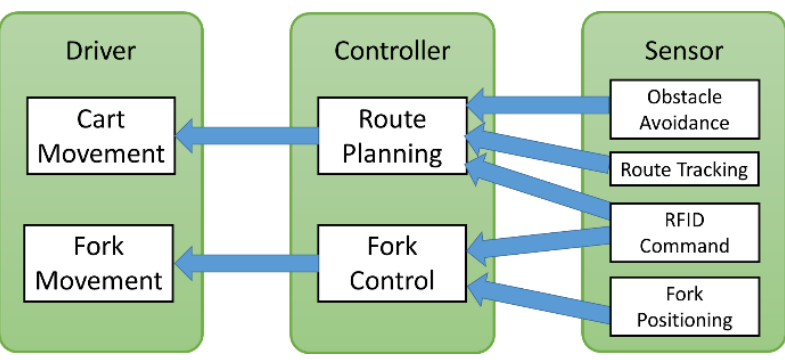

Fig. 2. System architecture of the auto-stacking warehouse truck.

\section{Simulation}

The scenery of our simulation includes a shipping area (also the packing area) and two warehouse area. The warehouse truck will be commanded to take the goods from factory packing area to the correct location of the warehouse and to leave the goods in the correct storage counter or to take the correct good from the storage counter of the warehouse to the shipping area. The goods can be stacked in the designated warehouse. For simplicity, the warehouse truck moves under the guide of a marked curve on the floor. To distinguish different warehouse, we marked a colored tag on the track. The whole simulation scenery is shown in Figure 3.

Our experimental truck is based on aluminum extruded material as a skeleton. A microchip 16-Bits MCU is used as the kernel and an Arduino chip is supplemented to control the truck. An infrared trace sensor is used to detect the route planned in advance, so that the truck can follow the route. A color sensor is used to read different storage areas. Truck moving and the fork moving up and down is driven by a 12 VDC deceleration motor. The fork 
stretching is to driven by a 5VDC stepper motor. Figure 4 shows our experimental auto-stacking warehouse truck. The truck is initially in the packing area. We use an RFID card to command the truck to pick up the goods and take it to the designated storage counter. The goods will be stored in a stacked manner.

There are two function buttons and an emergency stop switch on the truck, with the green button for picking goods up from the warehouse area to the shipping area, and the yellow one for delivery goods to the warehouse area for stack. The different warehouses are distinguished by two RFID cards. There is a stacking limitation in each warehouse area. The truck can move under the command of pushed button and the read RFID message. Once the destination warehouse is full of goods and a goods is planned to be stacked into, the warning light will be bright. The alert can be released by changing the command mode. In our simulation, the goods can be smoothly transported from the packing area to the storage area for stacking, or picked up from the storage area and transported to the shipping area. Figure 5 shows the scenario that a goods is in transportation to the warehouse area A.

\section{Discussion and Conclusion}

In this paper, we have made a brief discussion of the advantages of an unmanned warehouse. In order to improve the space efficiency of the limited storage area, stacked storage of goods is a good idea. In order to realize such a warehouse concept, we designed a simulated warehouse truck with function of auto-stacking. It can move on the specified route and store the goods in a stacked way to the designated area. When the stack area is full, it can alert the operator to change his instructions. This idea can be realized in a real warehouse area with cubic packed goods such as televisions, refrigerators, or air conditioners.

\section{References}

1. V. Giordano, J.B. Zhang, D. Naso and F. Lewis, Integrated supervisory and operational control of a warehouse with a matrix-based approach, IEEE Trans. Automation Sci. and Engr. 5(1) (2008) $53-70$

2. Z. Xie, W. He, L. Lv and J. He, The implement of warehouse management system based on RFID and

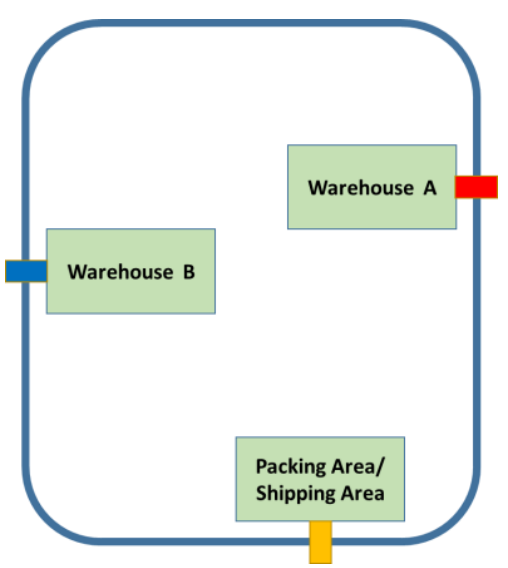

Fig. 3. Simulation Scenery.

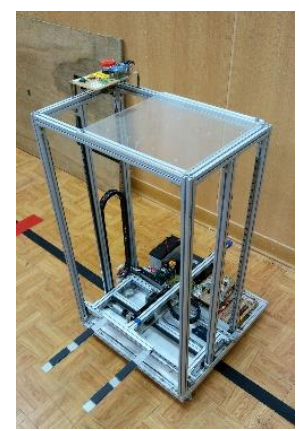

Fig. 4. Our experimental auto-stacking warehouse truck.

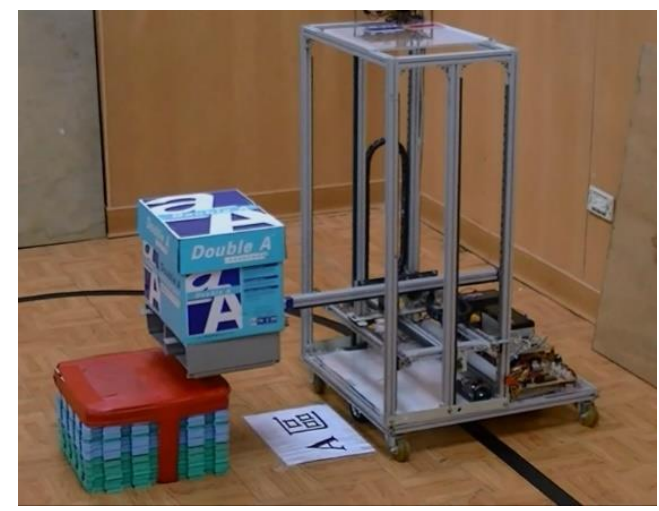

Fig. 5. A snap of the warehouse truck working.

wireless sensor network, IET Int'l Conf. on Wireless Sensor Network 2010 (2010) 98-103.

3. S. Adinandra, J. Caarls, D. Kostić, J. Verriet and H. Nijmeijer, Flexible transportation in warehouses, in: Automation in Warehouse Development (Springer, London, 2012). 
4. H. Wang, T. Cheng, and Z.A. Wu, Research on positioning algorithm of forklift-mounted RFID reader, Sensors \& Transducers 174(7) (2014)164.

5. S. Shead, Amazon now has 45,000 robots in its warehouses, Business Insider, UK, Jan. 2017. Available at: http://uk.businessinsider.com/amazons-robot-army-hasgrown-by-50-2017-1 